Migrations, transnationalisme et diaspora : théorie et études de cas

\title{
María Eugenia ANGUIANO y Ana María LÓPEZ SALA, Migraciones y fronteras. Nuevos contornos para la movilidad internacional
}

Lucile Medina

\section{(2) OpenEdition}

Édition électronique

URL : https://journals.openedition.org/remi/5717

DOI : $10.4000 /$ remi. 5717

ISSN : $1777-5418$

Éditeur

Université de Poitiers

Édition imprimée

Date de publication : 1 mars 2012

Pagination : 179-182

ISBN : 979-10-90426-03-0

ISSN : 0765-0752

\section{Référence électronique}

Lucile Medina, «María Eugenia ANGUIANO y Ana María LÓPEZ SALA, Migraciones y fronteras. Nuevos contornos para la movilidad internacional », Revue européenne des migrations internationales [En ligne], vol. 28 - $n^{\circ} 1$ | 2012, mis en ligne le 30 juillet 2012, consulté le 16 avril 2022. URL : http://

journals.openedition.org/remi/5717; DOI : https://doi.org/10.4000/remi.5717 


\section{NOTES DE LECTURE}

ANGUIANO, María Eugenia (ed.)

LÓPEZ SALA, Ana María (ed.)

Migraciones y fronteras. Nuevos contornos para la movilidad internacional. Barcelona : CIDOB, 2010. - $351 \mathrm{p}$.

ISBN : 978-84-92511-28-0

\section{I'ouvrage, coordonné par María Eugenia}

LAnguiano Téllez et Ana María López Sala, rassemble onze contributions en espagnol. Toutes se donnent pour objectif commun de croiser l'analyse des questions de frontières et de migrations sur les quatre continents.

Les deux coordinatrices nous offrent le fruit d'une belle coopération internationale, impulsée à travers le CSIC (Consejo Superior de Investigaciones Cientificas) espagnol et le COLEF (Colegio de la Frontera Norte) mexicain, deux institutions qui jouissent d'une reconnaissance académique internationale sur les migrations et les frontières. Il faut saluer cette entreprise par la difficulté à recueillir des données - à la fois en raison du caractère illégal des flux et de la sensibilité politique du thème dans nombre de pays - et par la difficulté à saisir la volatilité de ces flux. Travail de terrain et actualisation pointilleuse sont à la base de textes très documentés, étayés par de nombreuses références. Les bibliographies proposées renvoient à une littérature abondante sur le thème des migrations internationales, mais aussi à une connaissance des rapports de police, de patrouilles frontalières, de ministères, etc. ; elles témoignent de travaux approfondis de la part de chacun des auteurs et permettront au lecteur lui-même de poursuivre l'analyse. L'autre point d'intérêt est la diversité des espaces étudiés. Malgré une prédominance des frontières européennes, trois études de cas portent sur l'Amérique latine, une sur l'Afrique et une sur l'Asie. On regrette juste parfois de ne pouvoir visualiser les trajectoires et les frontières dans l'espace, deux cartes seulement étant présentes dans tout l'ouvrage.
L'introduction écrite par María Eugenia Anguiano Téllez donne le ton. Elle s'ouvre sur la préoccupation politique de premier plan dont la migration internationale est devenue l'objet. Elle cite Lelio Mármora (Las políticas de migraciones internacionales, OIM-Paidós, 2002), grand spécialiste des politiques migratoires, pour qui «les migrations ont cessé d'être un processus positif et désiré pour être visualisées de plus en plus comme un "problème", qui entre dans la catégorie de "conflit politico-social" "). Or, la migration internationale implique, par définition, le passage d'une ou plusieurs frontières nationales. $\dot{A}$ ce titre, il apparaît clairement que dans la dernière décennie du XXe siècle, la formulation de politiques migratoires et la gestion de la migration internationale ont connu une approche renouvelée. Étroitement liée au contrôle et à la militarisation des frontières, celle-ci est allée jusqu'à la construction de murs sur les lignes de division internationales. Cette tendance des pays d'accueil à considérer la porosité des frontières et la migration non autorisée comme des menaces pour la sécurité nationale a dérivé sur l'établissement de dispositifs de contentions frontalières et migratoires, appuyés par des technologies sophistiquées. Qu'il s'agisse de ces dispositifs ou des politiques migratoires de plus en plus restrictives, ils ont également affecté les conditions de la mobilité et les droits des personnes migrantes, même si le nombre de migrants hors de leur pays dépasse aujourd'hui les 200 millions.

Bien que les textes qui suivent cette introduction soient simplement numérotés de I à XI, la progression choisie correspond, à l'évidence, à plusieurs entrées dans le thème.

Les migrations internationales comme objet d'une préoccupation politique croissante

Les deux premières contributions de Jeroen Doomernik et Fabio Baggio plantent le décor en abordant de façon générale la croissante préoccupation des gouvernements en matière 
de migration internationale. Le premier analyse les politiques qui entendent limiter la réception des migrants et les types de contrôles externes (aux frontières à travers leur renforcement physique ou administratif, et en amont à travers l'octroi de visas) ou internes (permis de travail et de résidence, accès limité aux services de base, etc.). Le second se focalise davantage sur les frontières pour bâtir son analyse sur une typologie liée à la migration : frontières nationales (renforcement des frontières dont l'exemple le plus évident est la "fortification» des limites entre États : États-Unis-Mexique, Inde-Bengladesh, Thaillande-Malaisie, Ceuta et Melilla), frontières internalisées (mécanismes d'exclusion sociale des étrangers) et frontières externalisées (contrôles effectués dans les pays de transit ou même d'origine).

Externalisation, déterritorialisation et coopéra$\underline{\text { tion de la gestion des frontières }}$

Les quatre contributions suivantes s'intéressent à la consolidation de nouvelles frontières régionales autour de l'Union européenne et des États-Unis. Chacune des contributions montre la construction de périmètres de sécurité qui dans leur recherche d'efficacité, déplacent l'action de contrôle des frontières vers l'extérieur des territoires et également de manière croissante, vers l'intérieur de ces derniers. Ainsi prennent forme d'épaisses frontières, qui peuvent renvoyer le lecteur au concept de méta-frontière développé par Michel Foucher (L'obsession des frontières, Perrin, 2007). En effet, pour reprendre Ana María López Sala et Valeriano Esteban Sánchez, le nouveau locus du contrôle, qui rompt dans une certaine mesure la traditionnelle identification entre territoire et souveraineté, suppose une extension, un déploiement politique du contrôle migratoire, qui commence dans les pays d'origine, se poursuit dans les pays de transit, incorpore des actions sur la frontière et pénètre le territoire des pays récepteurs.

Chacune des contributions identifie également les routes d'entrée maritimes, terrestres ou aériennes, la géographie changeante, mais souvent l'unidirectionnalité des flux, et les stratégies déployées par les migrants pour contourner les barrières. Ana María López Sala et Valeriano Esteban Sánchez s'intéressent au renforcement des routes africaines et méditerranéennes vers l'Europe; Stefan Alscher se concentre sur le cas de la Pologne pour illustrer la très longue frontière orientale terrestre de l'Europe ; Ahmet Içduygu et Deniz Sert montrent une Turquie utilisée depuis les années 1980 comme pont vers l'Occident et devenue une importante route de la migration en transit ; enfin María Eugenia Anguiano Téllez s'attache à la complexité du Mexique, à la fois pays d'émigration, d'immigration et de transit, dont le territoire offre l'image d'une longue frontière verticale.

Ces quatre contributions s'attachent à montrer la pression que l'Union européenne et les États-Unis exercent sur les pays voisins pour retenir les vagues de migration "irrégulière » et l'enjeu diplomatique que cela représente, qu'il soit question des dispositifs mis en place par l'Union européenne pour surveiller les frontières du sud, ou " aider " la Turquie et les pays candidats de l'Est à améliorer leur gestion frontalière pour remplir les conditions de l'Acquis Communautaire ou du rôle des États-Unis dans la mise en place par le Mexique d'une Politique Migratoire Intégrale qui vise à la contention du flux de migrants illégaux sur les frontières guatémaltèque et bélizienne. Externalisation, déterritorialisation et coopération apparaissent ainsi à travers ces premières contributions comme les caractères récents de la gestion frontalière et migratoire.

\section{Migrations et territoires transfrontaliers}

Sans laisser de côté l'analyse des politiques migratoires, toujours présente en toile de fond explicative, les quatre contributions suivantes changent de prisme en privilégiant l'analyse régionale, à travers des cas de frontières où ancienneté et intensité des relations se répondent. C'est ici la manière dont les migrations et mobilités circulatoires façonnent des territoires transfrontaliers que les auteurs explorent, comme le résume le titre du chapitre de Susana Sassone et Geneviève Cortes, 
"Pasar y vivir la frontera ". Ce deuxième ensemble de contributions donne à voir le double visage des frontières : espaces de séparation politique et de contention migratoire, mais aussi de contact, de passage et d'intégration. C'est le cas d'abord de la frontière entre le Nicaragua et le Costa Rica étudiée par Abelardo Morales, connue pour sa circulation intense de travailleurs nicaraguayens. L'auteur s'attache à montrer l'existence d'une intégration fonctionnelle binationale et décortique le substrat des multiples interactions qui s'y jouent. S'intéressant à la migration bolivienne vers l'Argentine, Susana Sassone et Geneviève Cortes organisent leur réflexion selon trois axes : la frontière comme limite et espace de contrôle politique, la frontière comme lieu matériel du passage et la frontière comme objet de représentations liées aux pratiques et aux expériences des migrants. Elles soulignent que la frontière est avant tout, pour le migrant, le lieu où se déploient des stratégies d'usage particulières, le "savoir-traverser". Le lien avec la contribution suivante tient à la question clé $d u$ caractère légal/illégal de la migration. Blanca Garcés Mascareñas explore les confins de la légalité et de l'illégalité à travers l'exemple de la Malaisie où les travailleurs étrangers, perçus comme une compétition et une menace, sont soumis à un système coercitif de gestion de la main-d'œuvre immigrée. Elle ouvre un peu plus la réflexion sur la frontière en ne limitant pas celle-ci à sa réalité de limite géographique (le détroit de Malacca historiquement espace de circulation intense), mais en considérant les frontières internes de l'exclusion, qui limitent l'accès des étrangers à des espaces sociaux, politiques ou symboliques. Enfin, Tara Polzer et Akoko Akech mobilisent deux terrains frontaliers en Afrique du Sud (avec le Mozambique) et au Kenya (avec l'Ouganda), soumis à des défis similaires en matière d'immigration. Ils y montrent comment des populations transfrontalières socialement intégrées ne s'en trouvent pas moins légalement exclues dans l'accès aux services (eau, logement, enseignement, santé, etc.). Les auteurs montrent que la centralisation structurelle et les pratiques vont encore, dans les deux cas, à l'encontre de la capacité des acteurs du gouvernement local à incorporer dans la planification des services la réalité de la mobilité et de la présence de populations étrangères.

La dernière contribution est celle de Jorge Bustamante, éminent professeur mexicain de sociologie, expert en migrations auprès de l'ONU, qui théorise la question aiguë de la vulnérabilité des migrants. Convoquant Max Weber, il distingue vulnérabilité structurelle (dans la relation à l'État) et culturelle (socialement construite) et insiste sur l'asymétrie de pouvoir à la base de l'état de vulnérabilité.

Pour conclure, Ana María López Sala nous rappelle deux choses essentielles. La première est que nous vivons dans un monde qui, plus il est globalisé, plus il se trouve divisé et éloigné de l'idéalisme d'abolition des frontières. La seconde est que la vulnérabilité des migrants semble aller croissant, parallèlement à la considération grandissante de l'immigration comme une menace à la sécurité et à la mise en application de législations criminalisant l'immigration illégale.

María Eugenia Anguiano Téllez et Ana María López Sala ont réuni onze riches contributions - auxquels il faut ajouter une introduction et une conclusion nourries - qui toutes jouent parfaitement le jeu d'analyser les migrations au prisme des frontières, ou plutôt même les frontières au prisme des migrations. Il faut souligner que ce parti-pris est novateur. Ainsi, les recherches sur les migrations internationales se sont intéressées à l'analyse des champs migratoires, puis plus récemment aux dynamiques transnationales qui animent ces mouvements. Mais jusqu'à présent les frontières, bien que présentes par essence sur le parcours de ces migrations, sont demeurées comme inscrites en creux (on peut renvoyer cependant à certaines contributions de l'ouvrage dirigé par BIGO Didier, BOCCO Riccardo et PIERMAY Jean-Luc (Dir.) (2009) Frontières, marquages et disputes, Cultures \& Conflits, 73, 139 p.). $C$ 'est pour cela qu'il s'agit bien là d'une entreprise qui renouvelle l'approche de la question des migrations, mais aussi celle des frontières. La construction de l'ouvrage se fait en fonction 
de deux entrées : la plus classique, la frontière envisagée à travers sa fonction d'outil de contrôle dans la gestion de la migration; la seconde, la frontière regardée comme l'espace au cour de l'expérience migratoire, directement et particulièrement impacté par les mobilités. Le prisme des migrations permet de revisiter le concept de frontière à travers les notions d'internalisation et d'externalisation des frontières, complexifiant ainsi un peu plus la frontière en soulignant son caractère flou. Celle-ci est ainsi abordée dans ses deux dimensions de ligne et de territoire. Dans le premier cas, c'est la figure de l'État qui fixe l'attention, par le contrôle qu'il exerce aux frontières et les dispositifs qu'il met en place face à la migration. À travers le second prisme, c'est la figure du migrant qui s'impose. Et c'est justement un des grands intérêts de ce livre engagé de savoir mêler politiques migratoires et expériences des migrants, articles de l'acquis communautaire et récits des cayucos, et d'appeler de ses vœux une réflexion des gouvernements d'accueil sur le thème des migrations.

Lucile Medina

MCF en géographie

UMR ART-Dév, Université Montpellier 3

\section{BAROU, Jacques (dir.)}

De l'Afrique à la France. D'une génération à l'autre. - Paris : Armand Colin, Collection Sociétales, 2011. - 256 p.

ISBN : 9782200255848

Cet ouvrage vient enfin combler un - $m a n q u e$, et presque, pourrait-on dire, lever un tabou, concernant la vie quotidienne des immigrés originaires d'Afrique Noire en France aujourd'hui. En effet, depuis les ouvrages de Catherine Quiminal (1991), d'Albert Nicollet (1992), ou encore de Mahamet Timera (1996), les publications sur ce thème se sont faites rares. Le sujet est devenu sensible et les généralisations faciles, comme le montrent les récentes polémiques sur la délinquance des jeunes issus de l'immigration subsaharienne (Lagrange et Oberti, 2006) et sur la Condition noire en France (Pap Ndiaye, 2008). C'est pourquoi cette approche qualitative, initiée par la Caisse nationale d'assurance vieil- lesse (Cnav), et cette publication dirigée par l'anthropologue Jacques Barou, chargé de recherche au CNRS et spécialiste reconnu de l'immigration et de l'Afrique subsaharienne, est particulièrement bienvenue. Elle expose les résultats d'une enquête réalisée à partir d'entretiens auprès de vingt-deux familles migrantes originaires de treize pays d'Afrique Noire, analysés collectivement par une équipe de chercheurs en sociologie, anthropologie et démographie. L'enquête présente l'intérêt d'être intergénérationnelle puisque, dans chacune des familles, des représentants adultes de deux générations successives ont été consultés, ce qui permet de saisir les évolutions à l'œuvre en matière d'intégration, d'identité et de relation au pays d'origine. La publication en annexe des "synthèses familiales " concernant la biographie des personnes enquêtées, qu'il n'apparaît pas inutile de lire dans leur intégralité, donne un caractère particulièrement vivant et fondé aux analyses présentées dans l'ouvrage, qui se divise en sept chapitres.

Le premier, sous la plume de Jacques Barou, rappelle l'histoire de cette immigration et présente un bilan des statistiques produites par l'Insee entre 1999 et 2008 au sujet de la population originaire d'Afrique subsaharienne installée en France. Le second chapitre, du même auteur, concerne le traitement institutionnel de cette migration africaine par l'État français et son caractère monétariste et volontariste en matière de développement. Le troisième chapitre, écrit à trois mains par l'ethnologue Martine Segalen, Sabrina Aouici et Rémi Gallou, deux socio-démographes de la Cnav, part du constat que les modes de représentation de la famille sont différents en Afrique et en France. Ils analysent la façon dont les représentants des deux générations composent avec les deux cultures dans leurs manières de "faire famille ». Puis, l'ouvrage aborde la mobilité socio-professionnelle des migrants subsahariens et de leurs enfants à travers un chapitre co-écrit par Jacques Barou, Rémi Gallou, Sabrina Aouici et la sociologue Claudine Attias-Donfut. Le texte se divise en deux parties. La première vise à dresser, face au stéréotype bien connu de l'immigré 
africain illettré, le tableau d'une immigration " bimodale ", c'est-à-dire qui concerne à la fois des personnes sans aucune formation et d'autres d'un niveau d'études supérieur à la moyenne française. La deuxième porte utilement sur la " spirale déviante » qui a pu conduire certains jeunes issus de l'immigration subsaharienne et plus particulièrement sahélienne sur les voies de la délinquance ou de l'échec scolaire. Les auteurs reviennent sur la diversité des modèles familiaux en Afrique en distinguant en particulier les sociétés matrilinéaires et patrilinéaires. Originaires de ces dernières, les migrants élevés dans le cadre de la famille élargie avec des rôles codifiés assignés à chacun, et notamment l'autorité absolue au patriarche, ne retrouvent pas en France les modèles permettant de légitimer leur propre autorité auprès de leurs enfants, et ont l'impression de perdre l'emprise sur eux, sans pour autant s'ouvrir au dialogue et au modèle français d'éducation. Cependant la très faible proportion de délinquants (un seul) dans les familles enquêtées par l'équipe de la Cnav, au sein d'un échantillon aléatoire ne visant pas forcément les quartiers sensibles, montre que le processus de concentration résidentielle est déterminant dans les phénomènes de délinquance. Claudine Attias-Donfut nous propose ensuite un article qui emprunte beaucoup à l'anthropologie, s'interrogeant sur le rapport à la vieillesse et à la mort des migrants originaires d'Afrique Noire. L'auteur souligne de manière très pertinente le dilemme, parfois existentiel, qui se pose aux migrants qui doivent choisir entre être inhumés en France auprès de leurs enfants ou en Afrique auprès des ancêtres. Le chapitre 6, écrit par Sabrina Aouici et Rémi Gallou, interroge des dynamiques identitaires chez les immigrés originaires d'Afrique Noire et leurs enfants nés en France. On sait que la notion d'identité a suscité des débats politiques en France en 2009 et 2010. Elle peut aussi poser des problèmes épistémologiques en sciences sociales (Laplantine, 1999) et ici les auteurs choisissent de la comprendre comme une construction liée à l'idée de citoyenneté et d'appartenance. L'article, qui s'appuie abondamment sur des entretiens issus de l'enquête, montre finalement l'extrême hétérogénéité du sentiment d'appartenance chez les migrants subsahariens et leurs enfants et la difficulté qu'il peut y avoir en fonction des contextes à se sentir à la fois Noir et Français. Enfin, l'ouvrage se clôt sur un article écrit par Sabrina Aouici, Rémi Gallou et la socio-anthropologue Nicole Lapierre. Il fait le point sur les discriminations vécues par les enquêtés et de manière plus générale sur l'émergence récente de revendications propres à la communauté noire en France. On est passé depuis les années 2000 d'une simple affirmation de l'égalité républicaine, à la reconnaissance des diversités et enfin à l'affirmation des "minorités visibles». Les événements de 2005, à savoir successivement les incendies ayant coûté la vie à des immigrés africains particulièrement mal logés, au sujet desquels un procès s'ouvre d'ailleurs aujourd'hui, puis les émeutes dans les banlieues qui ont pu faire penser à une "colère noire" (Dupaire, 2007) à l'égard de ces mauvaises conditions de vie et d'intégration, ont constitué un point d'achoppement des débats. Comme le montre cet ouvrage collectif, les sciences sociales ont toute latitude de s'en saisir et de les enrichir de propos construits, fondés sur des réalités vécues et permettant ainsi de dépasser les préjugés.

On notera que deux enquêtes adoptant une méthodologie similaire au sujet des populations originaires d'Afrique subsaharienne installées au Royaume-Uni et en Afrique du Sud ont été menées en parallèle. Une comparaison plus approfondie des formes d'intégration dans ces différents pays devrait donner lieu à une publication ultérieure que nous attendons avec impatience, pour mieux saisir la spécificité française et européenne dans le traitement des minorités noires.

Jeanne Semin CEMAf-Ivry 